Less is More II: an optimistic view of piecewise smooth bifurcation theory

Glendinning, Paul

2016

MIMS EPrint: 2016.19

Manchester Institute for Mathematical Sciences

School of Mathematics

The University of Manchester

\footnotetext{
Reports available from: http://eprints.maths.manchester.ac.uk/

And by contacting: The MIMS Secretary

School of Mathematics

The University of Manchester

Manchester, M13 9PL, UK
} 


\title{
Less is More II: an optimistic view of piecewise smooth bifurcation theory
}

\author{
Paul Glendinning \\ School of Mathematics, University of Manchester, Oxford Road, Manchester M13 9PL, U.K. \\ p.a.glendinning@manchester.ac.uk \\ I am grateful to Mike Jeffrey, Rachel Kuske and David Simpson for conversations that helped crystallize these \\ ideas, and to the Simon Foundation for support at the CRM, Barcelona.
}

\begin{abstract}
The analysis of piecewise smooth bifurcations reveals an alarming proliferation of cases as the dimension of phase space increases. Rather than attempt the derivation of exhaustive lists of possibilities we describe ways of giving less detailed, but possibly more useful, results.
\end{abstract}

\section{Introduction}

"Take some more tea," the March Hare said to Alice, very earnestly. "I've had nothing yet," Alice replied in an offended tone, "so I can't take more."

"You mean you can't take less," said the Hatter: "it's very easy to take more than nothing." [2] chapter 7.

Mathematicians often aim to produce classification theorems, and normally these attempt to be as complete as possible. However, as argued in [4], the number of bifurcations in piecewise smooth (PWS) systems increases alarmingly with the dimension of the ambient phase space or the complexity of the system, and this may mean that complete descriptions, in the same spirit as would be given for smooth systems, become infeasible and certainly become unwieldy. This creates a problem for mathematicians with a background in smooth bifurcation theory: there are many potentially beautiful problems such as the existence of Shilnikov homoclinic bifurcations with sliding segments in local bifurcations of stationary points of PWS systems [4], but if the general result is that for the boundary equilibrium bifurcation in $\mathbb{R}^{n}$ then the local dynamics can contain analogues of any bifurcation of smooth systems in $\mathbb{R}^{n}$, as may well be the case, then it is unclear how to proceed.

This dilemma suggests that mathematicians should find coarser, but generally useful, statements about the local bifurcation structure of PWS system and provide a general framework or set of techniques which researchers interested in applications can use on particular examples. Thus the theoretician might need to rein in his or her natural inclination towards a detailed classification and provide instead descriptions that are less complete but easier to follow and interpret. Or again describe some things that cannot happen (as so much can). In this paper we give some examples of results that fit into this 'less is more' way of seeing the dynamics of PWS systems. 


\section{PWS maps of the interval}

There are a number of results describing the dynamics of PWS maps based on the ideas of Milnor and Thurston [5], which was circulating in preprint form from 1977. However, these results depend on a knowledge of kneading theory, an algebraic version of symbolic dynamics, and this means the proofs may seem abstruse, and a great deal of information is implicit in an algebraic invariant (the kneading invariant) which characterizes the nonwandering set of a map. (A point $x$ is wandering if there exists an open neighbourhood $U$ of $x$ such that $f^{n}(U) \cap U=\emptyset$ for all $n>0$, and a point is non-wandering if it is not wandering.) A weaker version of their theorems can be proved without recourse to new formalism. This simplified version is an example of the 'less is more' approach: the result is general, but for any example more work would be needed to add greater precision to statements. As is standard in the theory of maps of the interval there is an issue about the existence of homtervals. These are open intervals $J$ on which $f^{n} \mid J$ is a homeomorphism for all $n=0,1,2, \ldots$. The basin of attraction of a stable periodic orbit may contain homtervals, and homtervals that are not in the basin of attraction of a periodic orbit are called wandering intervals.

The dynamics is described in terms of Markov partitions and Markov graphs. A Markov partition is a union of closed sets that are permuted by the map and hence the images of elements in their complement, $\left(L_{i}\right)$, are either disjoint, $f\left(L_{i}\right) \cap L_{j}=\emptyset$, or $L_{j} \subseteq f\left(L_{i}\right)$. This means that a Markov graph can be defined with vertices labelling the connected elements of the complement and a directed edge from $i$ to $j$ if $L_{j} \subseteq f\left(L_{i}\right)$. Given any (finite or infinite) path allowed by this graph then there exists a point that passes through the sets in the order described by the path.

Theorem 1 Suppose that $f: I \rightarrow I$ is a PWS map with two continuous monotonic branches and a single critical point or point of discontinuity. Then there exists $0 \leq n \leq \infty$ such that the nonwandering set can be written as a union (disjoint except possibly $T_{n-1}$ and $A_{n}$ )

$$
A_{n} \cup\left(\cup_{0}^{n-1} T_{k}\right)
$$

where dynamics in $T_{k}$ is determined by a finite Markov graph (possibly zero entropy) and $A_{n}$ is (up to homtervals) a union of periodic orbits or a union of intervals if $n<\infty$ or a Cantor set if $n=\infty$.

We will sketch a proof in the case that the map is differentiable and expanding away from the critical point or the point of discontinuity, which will be denoted by $c$. This is Lemma 2 below. The proof relies on the idea of induced maps and renormalization. If $c \in J$ write $J=J_{0} \cup\{c\} \cup J_{1}$ where $J_{0}=J \cap\{x<c\}$ and $J_{1}=J \cap\{x>c\}$.

A map $f$ is renormalizable if there exists $J$ with $c \in J$ and positive $n_{0}$ and $n_{1}$ with $n_{0}+n_{1}>2$ such that $f^{n_{k}} \mid J_{k}, k=0,1$, is a homeomorphism and

$$
f^{n_{0}}\left(J_{0}\right) \cup f^{n_{1}}\left(J_{1}\right) \subseteq J .
$$

If $f$ is renormalizable then the induced map $F: J \rightarrow J$ defined by $F(x)=f^{n_{k}}(x)$ if $x \in J_{k}, k=0,1$ is again a map with a single discontinuity or critical point.

Finally, $f: I \rightarrow I$ is transitive if for all open $J \in I$ there exists $n$ such that $I=$ $\cup_{0}^{n} c \ell\left(f^{k}(J)\right)$.

Lemma 2 If $f: I \rightarrow I$ with I smallest such interval and $\left|f^{\prime}(x)\right| \geq a>1$ if $x \neq c$, then either $f$ is renormalizable or $f$ is transitive. 
Where does this get us? If $f$ is transitive then the non-wandering set is $I$. If $f$ is renormalizable then the components of the set

$$
K=\left(\bigcup_{k=0}^{n_{0}-1} f^{k}\left(J_{0}\right)\right) \cup\left(\bigcup_{r=0}^{n_{1}-1} f^{r}\left(J_{1}\right)\right)
$$

are permuted by the map so the complement $I \backslash K$ is a union of closed intervals (possibly trivial or even empty) $L_{k}$ such that either $f\left(L_{i}\right) \cap L_{j}=\emptyset$ or $L_{j} \subseteq f\left(L_{i}\right)$ - the condition for a Markov graph. So the dynamics is divided into the dynamics in the sets $L_{k}$ which is determined by a finite Markov graph and the dynamics induced by the renormalized map (again a two monotonic branch map) in $K$.

Sketch proof of Lemma 2: Intervals expand under iteration so images of any open interval $V$ must eventually intersect $c$. Call this image $V_{0}$. $V_{0}$ is divided into two by $c$ and each component will also return for the first time. Either these returns are are inside $V_{0}$ (so $f$ is renormalizable) or define $V_{1}$ to be the union of $V_{0}$ and its first returns. Repeat and note that each return is after the same or a shorter number of iterations and hence either $f$ is renormalizable or a larger interval $V_{2}$ can be constructed from $V_{1}$ and its returns. If $f$ is not renormalizable then for every interval the process never stops and the return times tend to a limit and the sets tend to a limit, $V_{\infty}$. If the sum of the limiting return times is greater than one then $f$ is renormalizable on $V_{\infty}$ (a contradiction), otherwise return times are 1 (and this is achieved in finite time) and since $I$ was minimal $V_{\infty}=I$ and the map is transitive.

This is a simple way of describing the dynamics of all piecewise monotonic maps with a single discontinuity. It has some detail (finite Markov graphs) but leaves a lot unsaid, so it does not require sophisticated arguments: less is more.

\section{The border collision normal form: Young's Theorem}

Let $\mathbf{x}=\left(x_{1}, x_{2}\right)^{T}$, then the border collision normal form is a piecewise affine map of the plane:

$$
\mathbf{x}_{n+1}=\left\{\begin{array}{ll}
A_{0} \mathbf{x}_{n}+\mathbf{m} & \text { if }\left(x_{1}\right)_{n} \leq 0 \\
A_{1} \mathbf{x}_{n}+\mathbf{m} & \text { if }\left(x_{1}\right)_{n} \geq 0
\end{array}, \quad \text { with } \quad A_{k}=\left(\begin{array}{cc}
t_{k} & 1 \\
-d_{k} & 0
\end{array}\right), k=0,1,\right.
$$

and $\mathbf{m}=\mu(1,0)^{T}$.

The parameter $\mu$ is considered to be the bifurcation parameter and some results for these maps are described in [4]. Banerjee et al. [1] show that the border collision normal form has parameters with (a) a trapping region; and (b) transverse intersections of stable and unstable manifolds and hence quasi-one-dimensional attractors: this has been called robust chaos. Young provided the tools to make these statements more precise [6].

Let $R=[0,1] \times[0,1]$ and let $S=\left\{a_{1}, \ldots, a_{k}\right\} \times[0,1]$ be a set of vertical switching surfaces with $0<a_{1}<\cdots<a_{k}<1$. Then $f: R \rightarrow R$ is a Young map if $f$ is continuous, $f$ and its inverse are $C^{2}$ on $R \backslash S$ and $f=\left(f_{1}, f_{2}\right)^{T}$ satisfies the expansion properties (H1)-(H3) below on $R \backslash S$.

$(H 1) \quad \inf \left\{\left(\left|\frac{\partial f_{1}}{\partial x}\right|-\left|\frac{\partial f_{1}}{\partial y}\right|\right)-\left(\left|\frac{\partial f_{2}}{\partial x}\right|-\left|\frac{\partial f_{2}}{\partial y}\right|\right)\right\} \geq 0$, 


$$
\begin{array}{r}
\inf \left(\left|\frac{\partial f_{1}}{\partial x}\right|-\left|\frac{\partial f_{1}}{\partial y}\right|\right)=u>1, \text { and } \\
\text { (H3) } \sup \left\{\left(\left|\frac{\partial f_{1}}{\partial y}\right|+\left|\frac{\partial f_{2}}{\partial y}\right|\right)\left(\left|\frac{\partial f_{1}}{\partial x}\right|-\left|\frac{\partial f_{1}}{\partial y}\right|\right)^{-2}\right\}<1 .
\end{array}
$$

Let $J a c(f)$ denote the Jacobian matrix of $f$ and recall that $u$ is defined in (H2).

Theorem 3 (Young's Theorem [6]) If $f$ is a Young map, $|J a c(f)|<1$ for $x \in R \backslash S$, and there exists $N \geq 1$ s.t. $u^{N}>2$ and if $N>1$ then $f^{k}(S) \cap S=\emptyset, 1 \leq k<N$, then $f$ has an invariant probability measure that has 'absolutely continuous conditional measures on unstable manifolds'.

The technical conclusion in quotation marks means that the invariant measure projects nicely onto one-dimensional horizontal lines.

Remark 1: The theorem holds for $C^{2}$ functions so provided perturbations of the normal form are $C^{2}$ in phase space and $C^{1}$ close in parameters then conditions for the theorem will still hold (if they hold in the first place) and so behaviour is robust.

Remark 2: The theorem as actually stated in [6] has $u^{N}>2$ and $f^{k}(S) \cap S=\emptyset$, $1 \leq k \leq N$. However, no extra conditions on images of $S$ are required if $N=1$ and if $N>1$ then the requirement is that $f^{N}$ has similar geometry on vertical strips, which only requires non-intersection up to the $(N-1)^{t h}$ iterate, so we are confident that Theorem 3 is what was intended $[\mathbf{3}]$.

The criteria for the theorem to hold are easy to verify numerically making it possible to determine regions on which Young's Theorem holds and compare these with theoretical bounds in $[\mathbf{1}]$, see $[\mathbf{3}]$ for details. The point about this result is that one could be tempted to provide further details such as the Hausdorff dimension of the support of the measure (the attractor), but that the statement that there is an attractor with an invariant measure having a nice one-dimensional projection gives the essential picture without overcomplicating the story: less is more.

\section{Conclusion}

The two results described here meet what I consider to be the 'less is more' criterion. They hold for a good range of models, they are informative, but there is much extra detail that they do not provide and they do not attempt a complete topological classifications. Given the hazards created by the proliferation of bifurcations in PWS systems outlined in $[4]$, we consider the existence of these results a cause for optimism, and they provide a template for the expression of further descriptions of PWS dynamics.

\section{References}

[1] S. Banerjee, J.A. Yorke and C. Grebogi (1998) Robust Chaos, Phys. Rev. Lett. 80 3049-3052.

[2] L. Carroll (1865) Alice's Adventures in Wonderland, Macmillan.

[3] P. Glendinning (2011) Invariant measures of the border collision normal form, preprint.

[4] P. Glendinning (2016) Less is More I: a pessimistic view of piecewise smooth bifurcation theory, this volume.

[5] J. Milnor and W. Thurston (1988) On iterated maps of the interval, in Dynamical systems, LNM 1342, 465-563, Springer, Berlin.

[6] L.S. Young (1985) Bowen-Ruelle Measures for Certain Piecewise Hyperbolic Maps, Trans. AMS 287 $41-48$. 